\title{
Home Energy Management System Based on Priority of devices
}

\author{
Ahmed S Abd Elwahed ${ }^{* a}$, AbdElhalim Zakry ${ }^{b}$, Ghaoda M Amer ${ }^{a}$, and Radwa M Tawfeek ${ }^{a}$ \\ ${ }^{a}$ Department of Electrical Engineering , Faculty of Engineering, Benha University, Benha , Egypt \\ ${ }^{b}$ Department of Electrical and communication, Faculty of Electrical Engineering , cairo, Egypt
}

*Corresponding Authros : Ahmed S Abd Elwahed [ahmeddesabryamin79@gmail.com]

\section{ARTICLE DATA}

\section{Article history: \\ Received 15 Oct 2021 \\ Revised 19 Jan 2022 \\ Accepted 02 Feb 2022 \\ Available online}

Keywords:

Home Energy Management

Smart Home

Demand Side Management

Energy Efficiency

Optimization

Simulation

\begin{abstract}
The rising number of home devices, as well as the differences in technology between them (smart and traditional), are the causes of the difficulty in balancing the use of energy in the home. Furthermore, increased demand for energy often leads to pressure on electricity networks and utilities, which leads to interruptions or disruptions of services caused by peak load. All these factors contribute to the adoption of energy management systems to control and reduce usage and automatically monitor the usage of home devices. When consumption is controlled, it is beneficial to customers and electricity companies when demand is reduced at peak times.This research will present a prototype for an energy management system to control and monitor the usage of devices in the home, and automatic control utilizing MATLAB simulation and implementation employing a Microcontroller to assess Demand Response.The suggested algorithm would manage consumption to reach the lowest usage possible to decrease the monthly bill, reduce peak load based on customer priority, and ensure that the consumption does not exceed the specified value. The algorithm's accuracy is demonstrated by several situations of consumption in the home, where the algorithm was able to lower consumption by up to $39.5 \%$ when compared to consumption without utilizing the suggested algorithm.
\end{abstract}

\section{Introduction}

The energy problem and one of the greatest challenges for utility companies are energy losses. There are some factors that contribute to energy waste or loss. One of these is poor social behavior, such as power theft from lampposts and excessive electricity usage. Furthermore, losses in transmission and supply accounted for up to $20 \%$ of total output last year, according to the report by the Ministry of Electricity and Renewable Energy. In addition, a lack of seriousness or not a good investment in dealing with renewable energy, whether it is wind or solar energy.

Figure 1 shows the distribution of electricity usage by sectors in Egypt [1]. It is clear from the figures that about $40 \%$ of the usage is used by the residential sector; Thus, the use of EMS at home may lead to optimal use and lead to reduced electricity consumption.
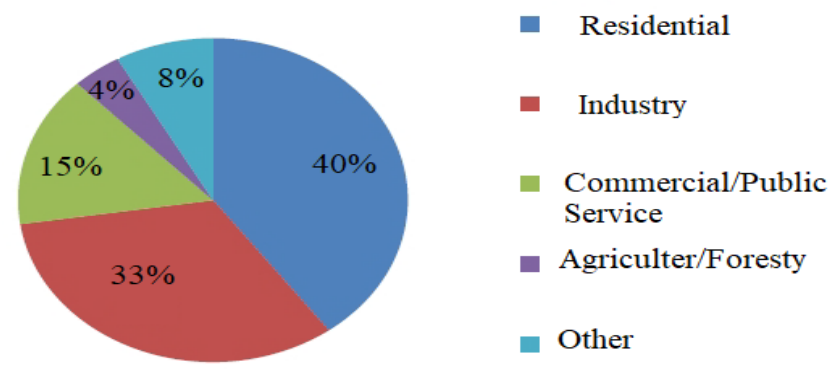

Figure 1: Egypt's electricity distribution by sectors.

\section{Literature Review}

This paper discusses the ways of optimizing energy usage and efficiency. Furthermore, there are methods of reducing the problems of frequent blackouts and power shortages. 
Energy Management Systems (EMS) believes that reducing energy usage and reducing peak load is good for all energy companies. This means that EMS is likely to be successful and fundamentally necessary in the near future.

The main objective of this research is to control the electricity consumption of appliances to reduce consumption and minimize bills. In addition, it reduces load peaks and improves power grid reliability.

Utility enterprises in developed countries have taken various measures to improve energy use and constrain consumption through planning and good connection between utilities, from product distribution to load distribution. There are several methods for both utility companies and users to use power more efficiently.

In terms of companies and utilities. They use a new approach based on network technologies, such as Demand Response Programs (DRP) [2], to motivate users to use the smart meter for optimizing the usage of energy, such as price, the 1-day-ahead price, Incremental Block Rate (IBR), Critical Pick Price (CPP), and Real-Time Price (RTP).

These techniques are important in order to establish a balance between the time-varying needs of customers [3], improve the performance of electrical devices, and enhance energy efficiency.

On the hand of users, they can decrease consumption through the use of applications or software programs like scheduling load in a home by the Energy Management Control System (EMCS) [4], applying schedule power using peak load shifting [5], or through scheduling home appliances using the Home Energy Management System (HEMS) by using one of the experimental techniques, whether it is the Flower Pollination Algorithm (FPA) or the Jaya Optimization Algorithm (JOA) [6], that decreases the rate of (PAR), wait time, and power consumption cost. Or, can apply home energy management according to that presented in [7], which depends on Harmony Enhanced Differential Evolution (HEDE), to enhance energy consumption flexibility and ensure demand does not exceed the limit at peak times.

The different techniques with the number of devices vary, such as traditional or smart devices. In addition, a lack of understanding of how to use contemporary technology is a barrier to the development of efficient EMS in Africa in general, and specifically in Egypt. Therefore, current standardization efforts are creating homogenization between devices to use HEMS technologies to overcome energy problems.

\section{Literature Review Elements of the problem and solution}

This section discusses the variables that influence energy use and result in energy loss or waste.

\subsection{The priority of the device is to decrease in-line losses}

The objective of employing the priority of the device in EMS is to control energy to reduce loss as well as control peak demand. Especially in cases of increased loads or power outages, some devices must be stopped or switched off to reduce the power load.

The load management unit can be used to delay or shift the peaks to another period. It not only reduces the total peak but also helps reduce the loss of the feeder and enhances the load factor [8].

Let's take an example to enhance the feeder's capacity. Two devices (c, d) carry the same loads that synchronize with the period of time $(\delta \mathrm{t})$ and the same resistance $(\mathrm{R})$ in the line. They are loaded into the line with resistance $(\mathrm{R})$, and the load currents (I) for each device are $\left(I_{c}\right.$ and $\left.I_{d}\right)$. Then the overall loss in the system at the moment as a result of the simultaneous peak's duration is:

$$
\begin{aligned}
\text { Total loss } & =\left(\mathrm{I}_{\mathrm{c}}+\mathrm{I}_{\mathrm{d}}\right)^{2} * \mathrm{R}^{*} \delta \mathrm{t} \\
& =\left(\mathrm{I}_{\mathrm{c}} \quad{ }^{2}+2 * \mathrm{I}_{\mathrm{c}} \quad{ }^{*} \mathrm{I}_{\mathrm{d}}+\mathrm{I}_{\mathrm{d}} \quad{ }^{2}\right) * \mathrm{R}^{*} \delta \mathrm{t}
\end{aligned}
$$

If peak loads are managed with the assistance of EMS to optimize consumption, no occurrences are allowed, as illustrated in Fig.2, then the losses become as:

$$
=\left(\mathrm{I}_{\mathrm{c}}^{2}+\mathrm{I}_{\mathrm{d}}^{2}\right) * \mathrm{R}^{*} \delta \mathrm{t}
$$

If they assume that both load currents are similar $\left(I_{c}=I_{d}=I\right)$, the overall loss from the first instance $(X)$ is:

$$
=4^{*} \mathrm{I}^{2 *} \mathrm{R}^{*} \delta \mathrm{t}
$$

In the second instance $(\mathrm{Y})$, the total loss becomes:

$$
=2^{*} \mathrm{I}^{2 *} \mathrm{R}^{*} \delta \mathrm{t}
$$
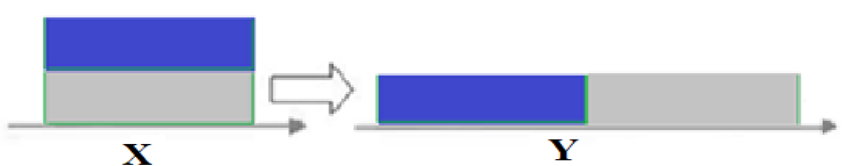

Figure 1: Different cases of in-line losses by using EMS. 
The value of the loss between the two instances is completely different. As a result, EMS is used not only to reduce losses, but also to improve and adapt the feed factor loading factor.

\subsection{Demand Response and Energy Efficiency} [9].

Both of them are closely related and connected to each other, whether it is Demand Response (DR) or energy efficiency (EE)

The term "Energy Efficiency" is described as the use of less energy while keeping the service at the same level. It means decreasing the value rate of energy consumed while delivering the same service or function by employing or replacing equipment with more efficient and reliable technology.

On the other hand, "Demand Response" (DR) is defined as changes in energy consumption as a result of incentives or pricing costs. The goal of DR was to relieve stress on the energy grid, particularly during periods of high load or prices, and maintain the reliability of the system. Customers who use the DR programs are encouraged to control their home appliances in an efficient and innovative way to minimize power consumption and reduce peak times.

\subsection{Types of appliances}

The devices may be classified into two categories:

1. Classify devices based on consumption of energy.

2. Classify devices based on modernization.

1. Classify devices based on energy consumption:

Each device has electricity consumption specifications [10], and they may be divided into three categories:

A. Baseline devices: These are devices, such as lighting, that usage energy nonstop with fixed energy consumption.

B. Burst devices: These are devices such as washing machines that use energy for a set period according to a program time.

C. Regular devices: These are devices that use energy in various states or stop working when a threshold is reached, according to temperature, time, or sensors like heaters and air conditioners.

2. Classify devices based on modernization:

A. Smart devices that can connect to the Internet for remote control via sensors and information communication technology (ICT) to perform an action or make a decision.

B. Traditional devices, which can not be operated or controlled remotely via the internet and are incompatible with energy management systems.

\section{Implementation of the proposed system for energy manegment}

The objective of this research is to use the EMS to turn used traditional devices at home into smart ones with high efficiency in order to regulate usage and improve energy consumption.

Most clients cannot proactively perform DR manually. As a result, the EMS is a way to automatically establish DR at home [11]. The suggested EMS algorithm takes into account the demand limit (DL) that is allowed to be used, the priority value for each device, and the value of the power consumed by each device, then calculates the Total Energy Consumption (TEC). The EMS can balance the consumption of devices by switching and shifting as needed, as shown in Fig. 3, with little impact on the customer's requirements or lifestyle [12].

\subsection{The algorithm strategy for energy management systems}

$\mathrm{C}$ The algorithm begins by gathering data about the status of each device, which is (ON/OFF), the set Demand Limit (DL), the set Load Priority (LP), and the entry of the usage Power of the device (P), to calculate the value of the usage energy for devices. The purpose of priority for devices is to make devices that have high priority with low power usage work first.

The algorithm's basic principle is to maintain the TEC rate and ensure that it doesn't exceed the DL. When consumption of total devices does not reach the DL, the device can be switched on. But when another device wants to turn on, the algorithm will return to the back loop to re-estimate and compute the TEC in order to maintain and ensure it is under the DL. This procedure is repeated for each of the remaining priorities $(1,2)$. 


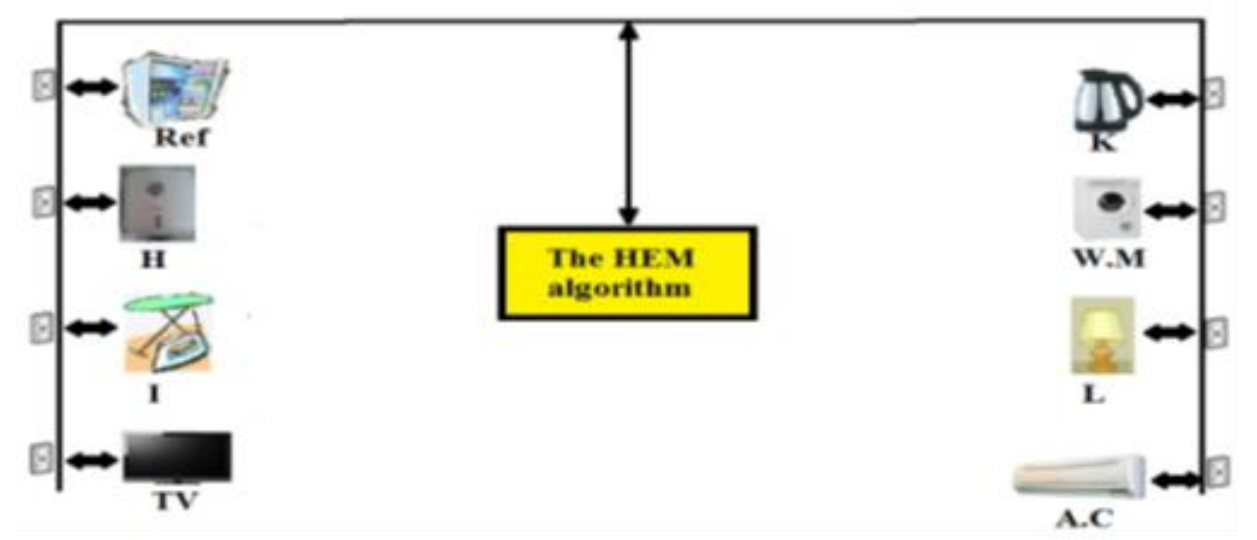

Figure 3: The EMS with devices at home.

ancer is a major public health concern in the world today and is responsible for one out of every six deaths worldwide, making it the second leading cause of death after cardiovascular disease [1]. Breast cancer is the most common type of cancer diagnosed in women around the world.

\subsection{Flowchart}

The first step in the algorithm in the flowchart in Fig.4 is identifying the priorities for devices and classifying them in order [13]. dividing them into three classes: the first class is the high priority, the second class is the medium priority, and the third class is the careless or low priority. Following that, in the second step, it arranges the devices in ascending order within each priority according to their power usage for each device.

When the algorithm is used, the algorithm starts and enables the devices that have priority (0) to turn on in sequence as long as the TEC does not approach the DL.

When the TEC approaches or equals the DL, it will loop back to look for changes in the values of the device's priority and consumption.

In another case, when the TEC exceeds the DL, then no device is permitted to run unless the TEC is recalculated using the algorithm by turning off other devices. This procedure is repeated for the remaining priorities $(1 \& 2)$ to guarantee that the total TEC does not increase more than the set DL.

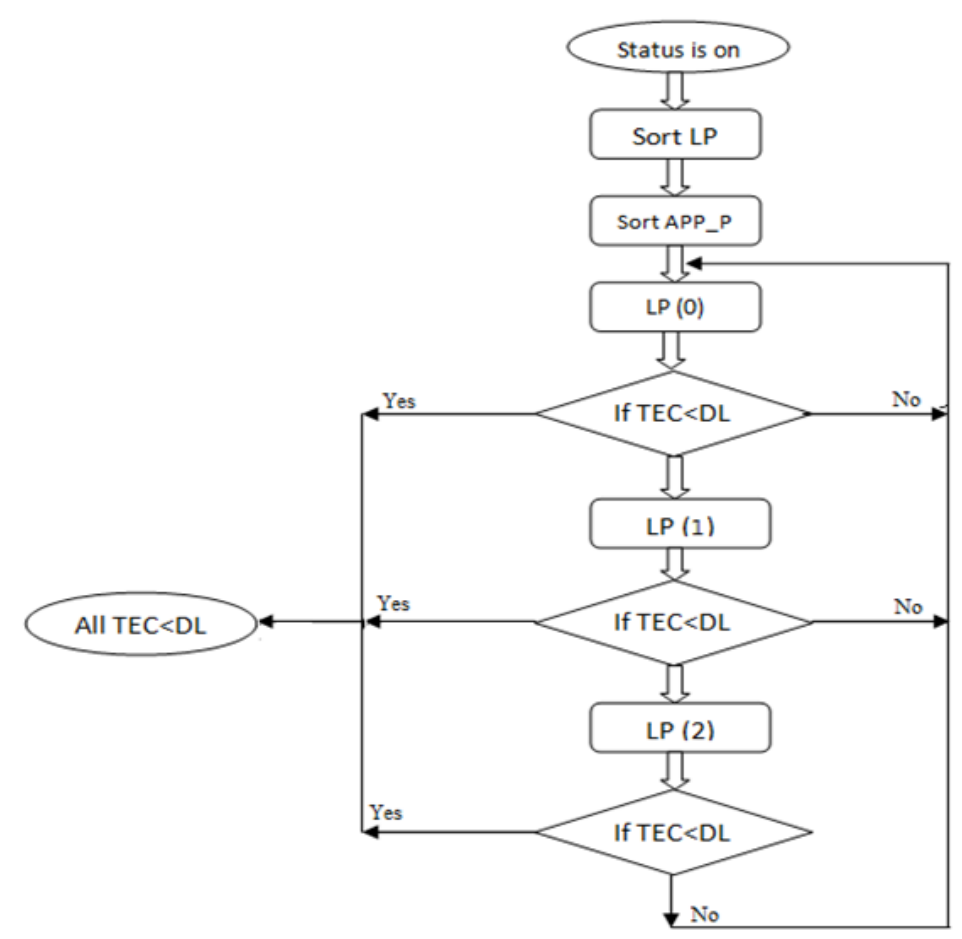

Figure 3: Flowchart of Energy Management System.. 
In another case, when two devices are equal in priority and must turn on at the same time, but the TEC will increase more than the DL, the flowchart arranges the devices' power in ascending order from lowest to highest, and allows the device that uses less power to turn on to keep the TEC below the DL.

The algorithm's benefit is that it ensures and keeps the TEC lower than the DL by turning on devices that have a higher priority with low power consumption. Therefore, it switches off the devices that consume a lot of power in order. Table 1 summarizes the suggested the devices in order of priority and power.

Table 1: Tthe devices in order of priority and power.

\begin{tabular}{|l|l|l|}
\hline Device & Priority (LP) & Power (P) \\
\hline TV & 0 & 90 \\
\hline Refrigerator & 0 & 350 \\
\hline Light & 0 & 350 \\
\hline Kettle & 1 & 1100 \\
\hline Heater & 1 & 1500 \\
\hline Air Condition & 1 & 1700 \\
\hline Iron & 2 & 1500 \\
\hline Wash Machine & 2 & 1800 \\
\hline
\end{tabular}

\subsection{Implementation of the energy management system}

For the implementation of EMS, the prototype was implemented in software and hardware. It was designed software through MATLAB as a control panel via a Graphical User Interface (GUI) that performs the calculation processing for the algorithm as shown in Figure 5. It is simple to use.
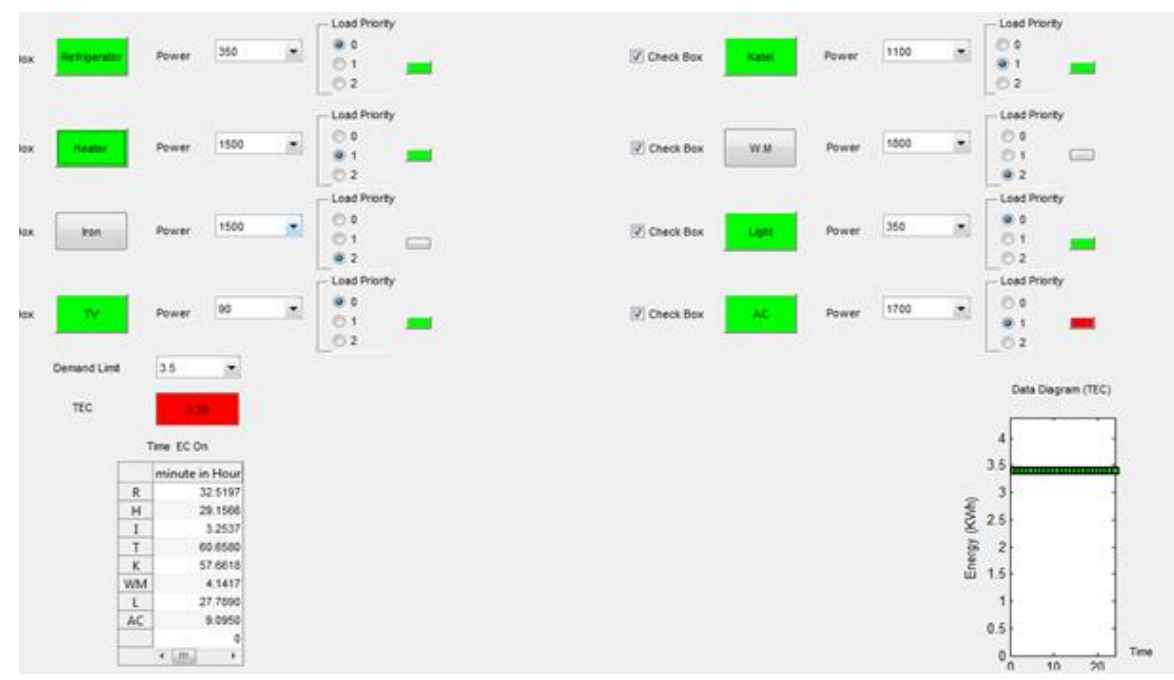

Figure 5: The control panel the energy management system is through a Graphical User Interface (GUI).

In the EMS hardware implementation, it created a kit of a module as a control unit to be used as an interface between the devices and the user. The module uses a microcontroller (PIC18F4550) and RS-232 [14]. The kit of a module is used as an interface between the MATLAB on the laptop and the devices. The RS232 is used to communicate between the control unit and the GUI on the laptop 


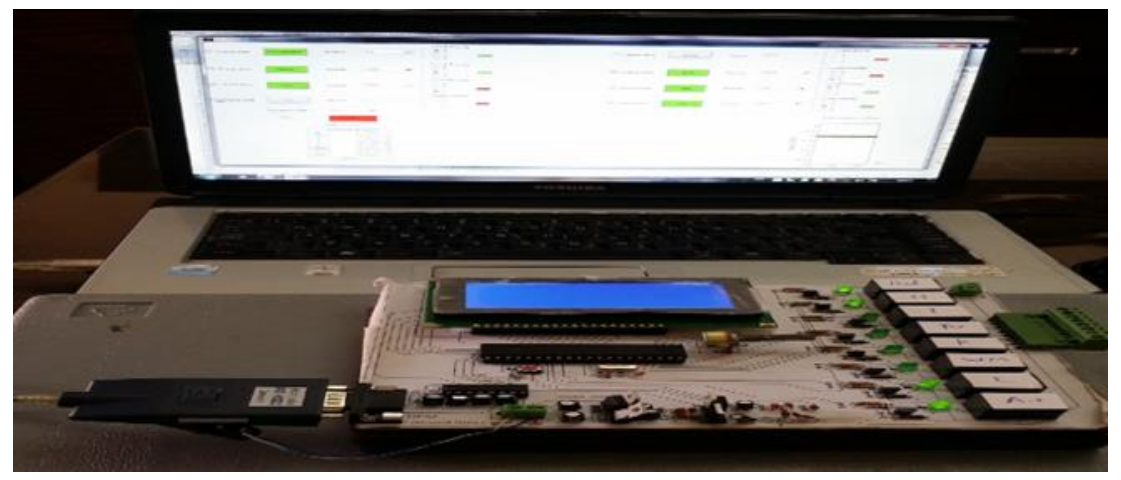

Figure 6: GUI and control unit as prototypes of the system

It is illustrated in Fig.6 that the integration of MATLAB and the module as a control unit of the implemented prototype of a system energy management, which is represented by the control unit and the GUI. The module as a control panel gets power from the device (P) and low power (LP) for each device that is turned on. Through communicating between MATLAB and the prototype of the control unit via RS 232, it takes the appropriate decision on whether to turn (ON/OFF) the devices.

\section{Experimental and Results}

In this section, will the experiment with EMS in some scenarios that have been subjected to the simulation, obtaining measurements and analyzing the results.

\subsection{The scenarios of the experiment}

This experiment includes most of the electrical appliances that are utilized in most households on a daily basis, like a refrigerator, a heater, a television, a kettle, a light, an iron, a washing machine, and an air conditioner.

It can estimate the average energy consumption of a regular home by calculating, for example, the average electricity usage of a family in Giza city over the week.

The user will choose the priority for these devices from low to high according to his desires and comfort, and then the classification of the devices into categories will be arranged devices in terms of power usage, from low-to-high in each category, as illustrated in Table. 1 as follows:

Devices with a high priority (0 value) are light, TV, and refrigerator. Devices with a medium priority (value 1$)$ are the AC, heater, and kettle. Finally, devices with a low priority (value 2) are the iron and the washing machine, because they are not used more than once or twice a week at most.

\subsection{The results of the simulation}

Using traditional household appliances can reduce electricity consumption and thus reduce the monthly bill by using EMS.

This is what was shown by the results of different scenarios through the use of EMS to manage the operation of traditional devices in the home efficiently and smoothly.

To show the enactment of the implemented EMS algorithm in controlling and reducing the energy in the home, three different cases are presented. Based on simulation results, it can be verified that using the EMS in any home can be more efficient and flexible. Simulations were used in three scenarios with real data from devices to reduce consumption and save money.

In the first scenario, the devices run and power consumption without any configuration or control by the EMS or not set the DL, as shown in the diagram when turning on devices without an algorithm in Figure 7
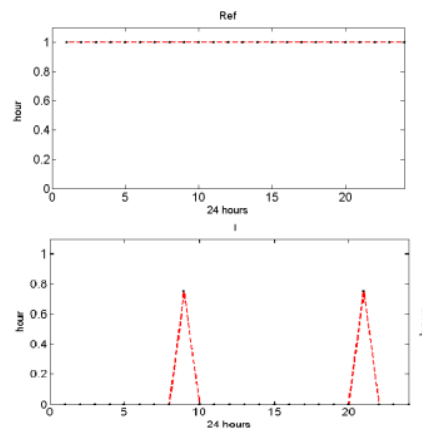

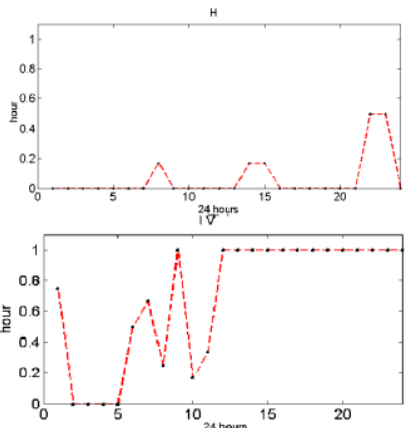

Figure 7: Simulation of the EMS on devices without DL.

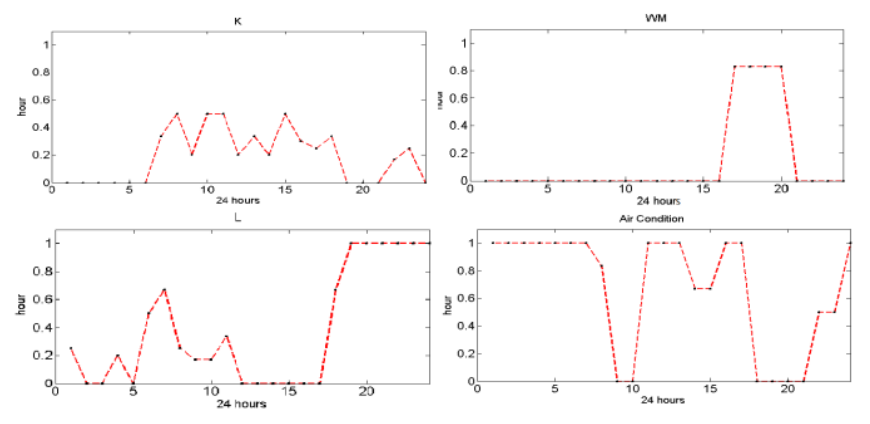
. 
Where there is no DL for EMS, the average consumption is $3.19 \mathrm{~kW} / \mathrm{h}$. It shows there are various load peaks in hours (14 to 21) due to a lack of management, which impacts the utilities' power, particularly during peak load, as seen in the diagram in Fig. 8.

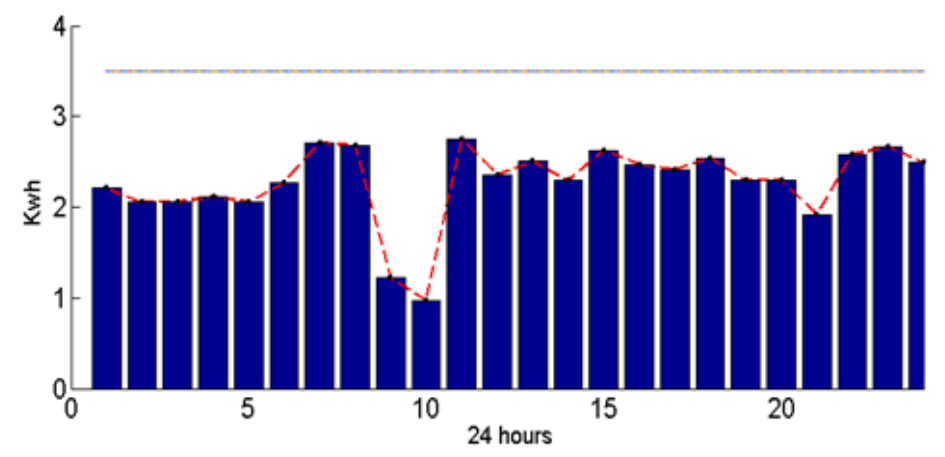

Figure 8: The rate of average consumption for devices using EMS without DL.

The largest average consumption from the continuous operation is for the A C. It reaches almost $40 \mathrm{~kW} / \mathrm{h}$ per day, as shown in Fig.9.

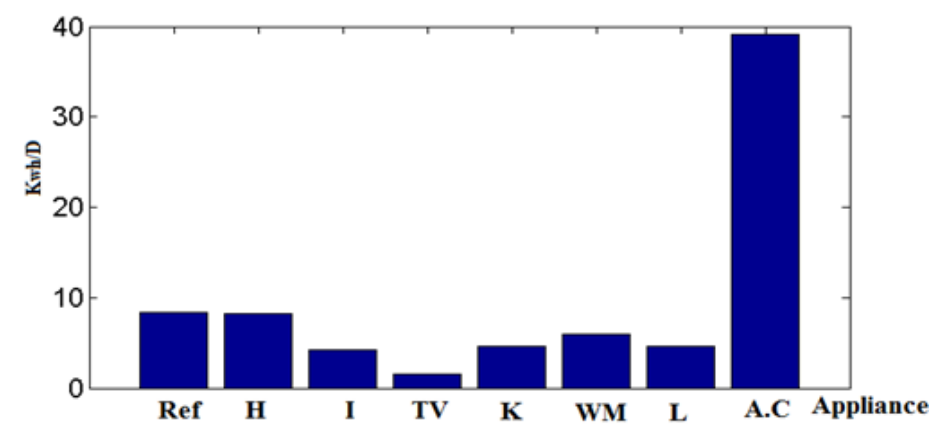

Figure 9: The TEC for each device per day using EMS without DL.

In the second scenario, apply the EMS at DL $=3.5 \mathrm{~kW} / \mathrm{h}$. The EMS system begins (ON/OFF) the various devices so that the TEC is no more than $3.5 \mathrm{~kW} / \mathrm{h}$, as illustrated in the diagram in Fig 10.
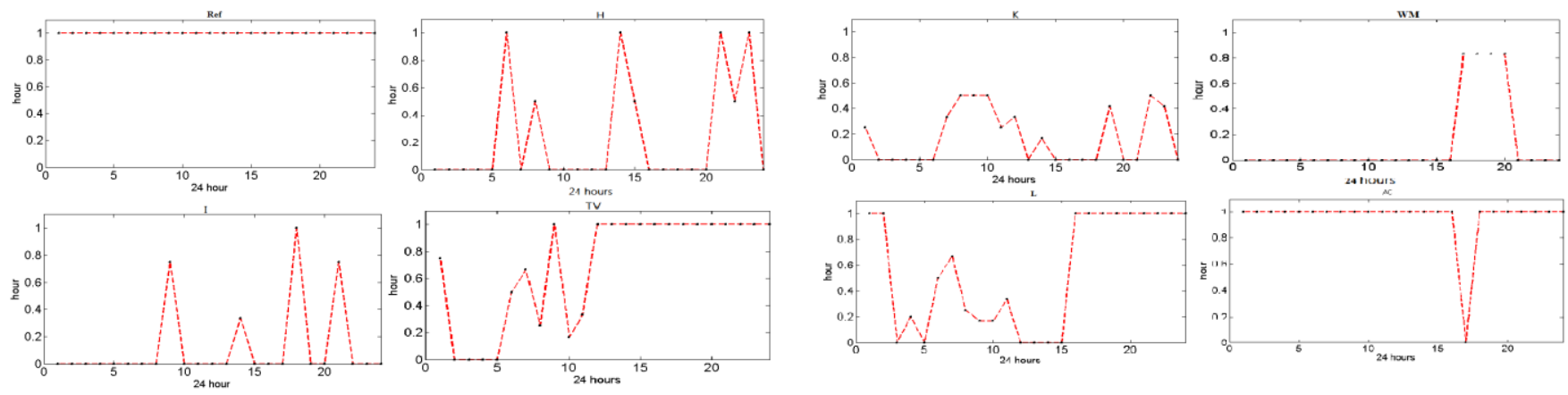

Figure 10: Simulation of the EMS on devices with DL $=3.5 \mathrm{~kW} / \mathrm{h}$.

Now the TEC is more adaptable and balanced. Furthermore, when compared to the first scenario, as shown in the first example, the load peak by using EMS displays various peaks and minimizes the TEC, as seen in Fig 11 . It should be noted that the TEC did not increase more than DL, and the energy consumption was minimized to $2.33 \mathrm{~kW} / \mathrm{h}$, which is less than $26 \%$ of the normal rate without utilizing the EMS. The peak consumption times during the day using DL $=3.5 \mathrm{~kW} / \mathrm{h}$ are shown in Fig 11 


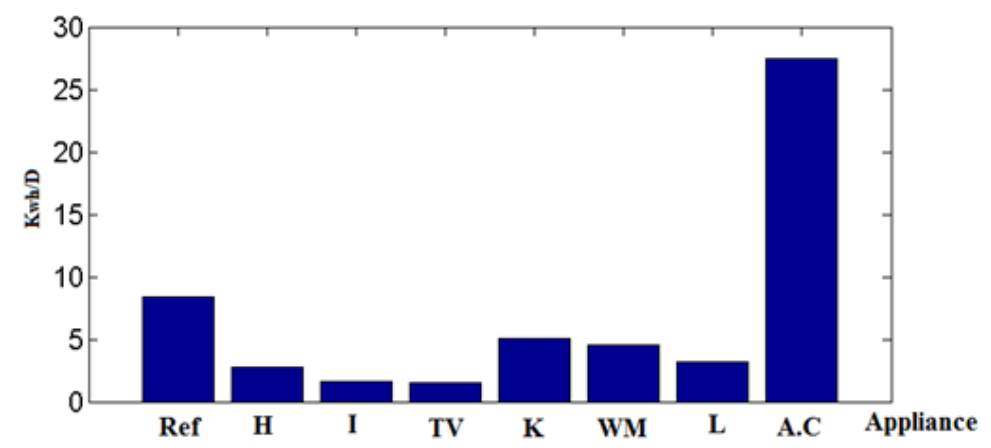

Figure 11: The rate of average consumption for devices using EMS with DL $=3.5 \mathrm{~kW} / \mathrm{h}$.

Note the impact on the rate of energy consumption, particularly those that consume large amounts of energy like heaters, irons, and air conditioners, compared to the previous scenario as demonstrated in the diagram in Fig 12.

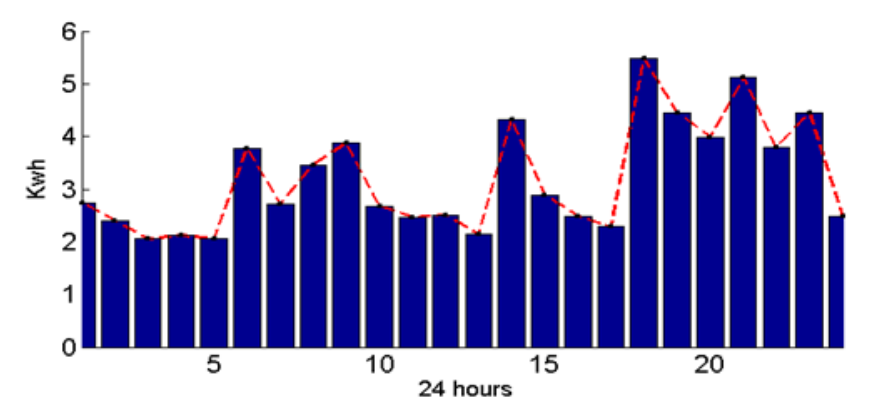

Figure 12: The TEC for each device per day with using EMS at DL=3.5 kW/h.

The last scenario is the third scenario when applying the EMS and setting the DL at $2.5 \mathrm{~kW} / \mathrm{h}$, as illustrated in the diagram when turning on devices with the EMS algorithm in Fig 13.
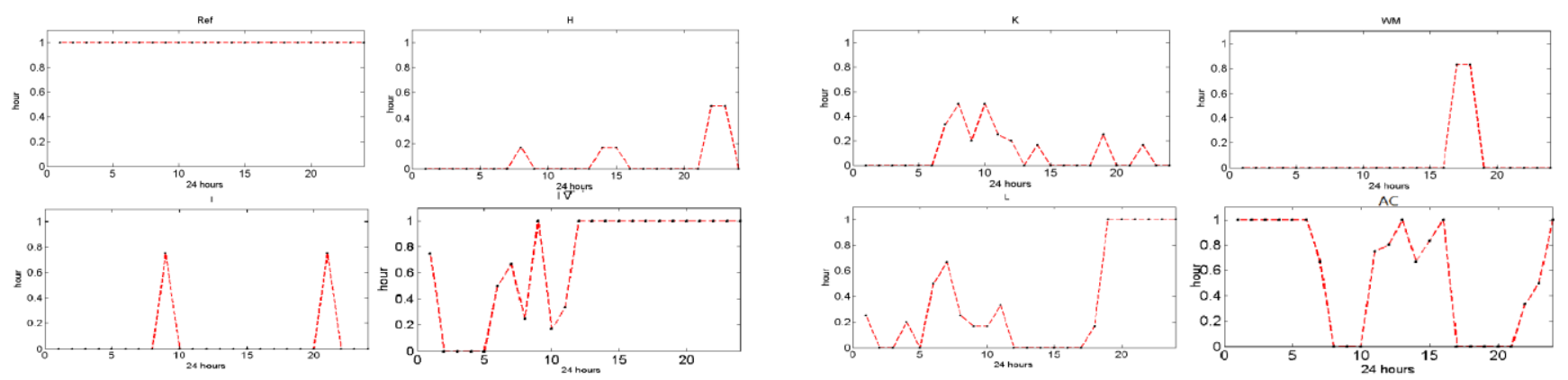

Figure 13: Simulation of the EMS on devices with $\mathrm{DL}=2.5 \mathrm{~kW} / \mathrm{h}$.

The peaks are more optimized during the day and ensured the average usage of energy was up to $1.92 \mathrm{~kW} / \mathrm{h}$ through the use of the EMS illustrated in Fig 14. The result is 39.5 percent, which is less than the rate of average in the first scenario without setting a DL.

The peaks are more optimized during the day and ensured the average consumption of energy was up to $1.92 \mathrm{~kW} / \mathrm{h}$ by using the algorithm illustrated in Fig 14. The devices consumption is reduced, which is up to 39.5 percent less compared to the average rate in the first scenario without the use of the EMS algorithm. 


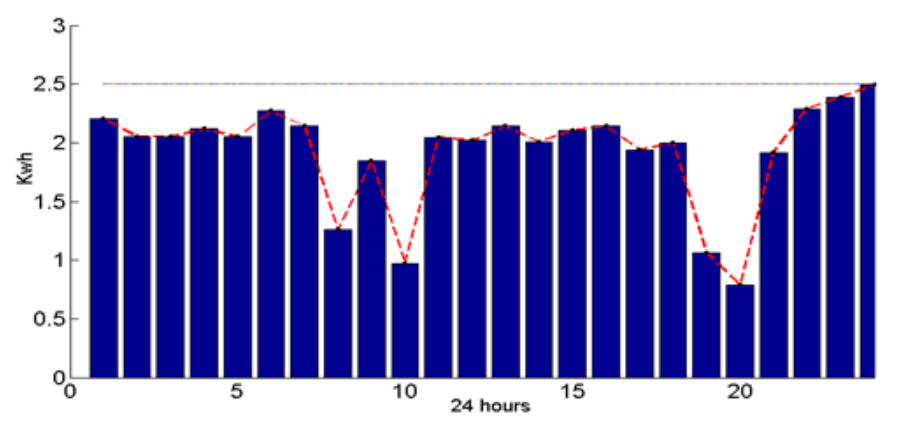

Figure 14: The rate of average consumption for devices using EMS with DL=2.5 kW/h.

As shown in Fig15, the rate of consumption per device during the day was reduced using the algorithm at $\mathrm{DL}=2.5 \mathrm{~kW} / \mathrm{h}$. It is evident in the consumption of A.C

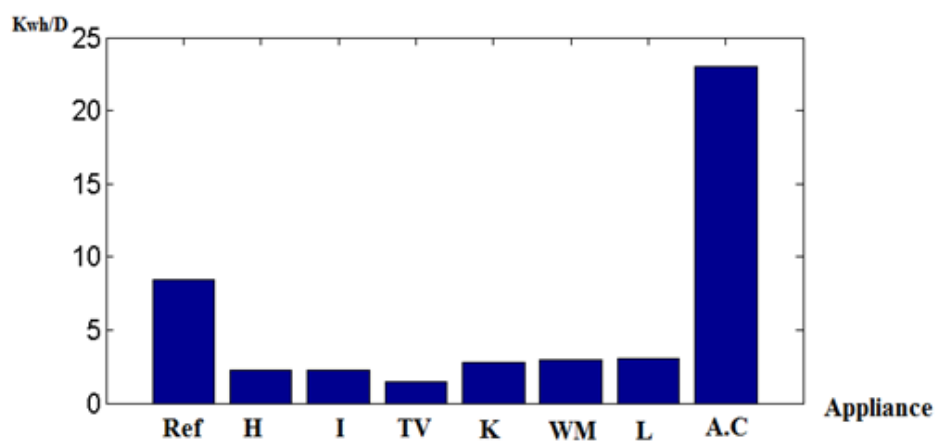

Figure 15: The TEC for each device per day with using EMS at DL $=2.5 \mathrm{~kW} / \mathrm{h}$.

\section{Analysis and Discussion of Results}

When applying EMS with devices in the home, it should take care of the following points:

1. The refrigerator is always turned on and is always connected to electricity, "which is typical."

2. The use of the A.C is always the highest of all devices. When not using the EMS to control it, the average usage of the A.C can reach $40 \mathrm{~kW} /$ day. When the $\mathrm{DL}$ is set to $3.5 \mathrm{~kW} / \mathrm{h}$, it can be reduced to around $28 \mathrm{~kW} / \mathrm{day}$, and it can be minimized to around $24 \mathrm{~kW} /$ day when the DL is set at $2.5 \mathrm{~kW} / \mathrm{h}$.

3. Burst devices, such as a W.M, turn on depending on a program or time of operation. They should not be stopped while the program is running or not completed. Stopping the device for any reason will result in restarting the program from starting or repeating some properties or features in it, such as re-heating the water. This leads to energy re-consumption and waste, which leads to an increased bill.

It is clear from comparing the data of the three scenarios that the average power usage is $(1.92,2.33$, and 3.19$) \mathrm{kW} / \mathrm{h}$ when the DL is set at (2.5 and 3.5) and without setting the DL, respectively. This resulted in a 39.5 percent reduction in average usage at $2.5 \mathrm{~kW} / \mathrm{h}$ and a 26 percent reduction at $3.5 \mathrm{~kW} / \mathrm{h}$. Also achieves a lower average A.C usage of $40 \mathrm{kWh} / \mathrm{d}$ and (28, then 23) $\mathrm{kWh} / \mathrm{D}$.

It leads to numerous benefits, including a decrease in the bill, more comfort, and an increased luxury level for users. In addition, enhance the service offered by utility companies, which solves the problem of electricity outages due to excessive loads, verifies the minimum cost, ensures reliability for utilities, improves energy efficiency, and decreases load peak times.

There are two points to keep in mind when using or applying the EMS:

A. The EMS has been shown to be able to maintain a TEC level below the DL. However, when a DL is small or low, it can compromise some luxury or cause some discomfort. For example, the low DL can cause the A.C to not turn on and thus increase the room temperature.

B. The DL should be set sensibly for a home in order to provide flexibility of use and the ability to use most appliances without compromising the convenience or inconvenience of the consumer. So that it does not cause energy reconsumption or wastage, and since this happens when the devices are restarted when the EMS allows them to work, the customer should select the minimum level of a DL that can be used with as many devices as possible. For example, reheating the water inside the washing machine after stopping.

As a result, while determining the minimum level of set DL value, the following factors must be considered:

- $\quad$ Determining priorities. 
- The DR's start and duration.

- Using a Variety of Patterns.

- Levels of comfort and luxury.

- Types of devices and value consumption (in $\mathrm{kW} / \mathrm{h}$ ).

Finally, this research presents the EMS and its impact on device management with the aim of reducing consumption at home without violating the customer's comfort and satisfaction. The EMS includes the setting of DL, device status (ON/OFF), device priority, the value of power for the device, duration of operation, and calculation of the TEC. To validate the EMS seriousness, the results are compared with the results of the previous search [6], which depend on the operation of MATLAB. As a result, neutrality in the simulation and in comparing the results is critical.The study's [6] depends on scheduling the smart devices inhome. The results show that the proposed HEDE method exceeded and overcome the other methods in terms of minimizing cost, which came up to $(24.668,23.277$, and $24 \%$ ) with HSA and EDE, respectively, which limit the client's use of the appliances at certain times and for a specified duration of time.In relation to the EMS that was applied in this paper, which was determined by the priority, the reduction in consumption reached $39.5 \%$, and therefore the reduction in usage is greater than the method in the study [6].This provides for increased consumer comfort without committing to set appliance operating times, making it more suitable for the reality of Egyptian society. Furthermore, the domestic devices used in this study are traditional and unintelligent.

\section{Conclusions and future works}

The suggested EMS may operate proactively and effectively in managing the run of the devices, although traditional devices may not be able to maintain the TEC level under the setting of the DL. The consumption can be reduced by up to 39.5 percent by using the EMS.

Simulation shows in scenarios that the results of energy usage based on the priority of the devices under the value of DL are more adaptable and optimized as a consequence of the energy savings achieved by switching off the devices.

This paper's analyses demonstrated the amount of comfort and luxury for consumers or lifestyles based on a setting DL. These studies and results are beneficial for energy companies and facility companies. They provide information in the home sector to know the difficulties of DR in decreasing frequent outages and electricity shortages and to minimize the high peak loads as much as possible.

In future work, it could be developed to achieve greater user satisfaction, by connecting to the Internet and applying new technology, which is the Internet of Things (IoT), and automatic interaction to detect and turn off unwanted devices. Moreover, automatic reporting of energy usage rates via smart meters for companies and electricity utilities.

\section{References}

[1] Annual report of the Egyptian Electricity Holding Company (EEHC) $2018-2019$. www.moee.gov.eg/english_new/report.aspx.

[2] Mohammad Shakeri and Nowshad Amin, "Transformation of conventional houses to smart homes by adopting demand response program in smart grid”, Smart Microgrids, pp 65-80, Intechopen, London, 2018, http://dx.doi.org/10.5772/intechopen.74780.

[3] S. Khan, Z. A. Khan, N. Javaid, S. M. Shuja, M. Abdullah, and A. Chand, "Energy efficient scheduling of smart home", vol. 927, no. march. springer international publishing, 2019, doi:10.1007/978-3-030-15035-8 7.

[4] N. Javaid, G. Hafeez, S. Iqbal, N. Alrajeh, M. S. Alabed, and M. Guizani, "Energy efficient integration of renewable energy sources in the smart grid for demand side management," ieee access, vol. 6, pp. 77077-77096, 2018, https://doi10.1109/access.2018.2866461.

[5] Elham Shirazi, Shahram Jadid. "Cost reduction and peak shaving through domestic load shifting and DERs". Energy, Elsevier pp. 124. 146e159, http://dx.doi.org/10.1016/j.energy.2017. 2017.

[6] Z. A. Khan, A. Zafar, S. Javaid, S. Aslam, M. H. Rahim, and N. Javaid, "Hybrid meta-heuristic optimization based home energy management system in smart grid," J. Ambient Intell. Humaniz. Computer. vol. 10, no. 12, pp. 4837-4853, 2019, https://doi.org/10.1007/s12652-018-01169-y.

[7] S. Kazmi, N. Javaid, M. J. Mughal, M. Akbar, S. H. Ahmed, and N. Alrajeh, "Towards optimization of metaheuristic algorithms for IoT enabled smart homes targeting balanced demand and supply of energy," IEEE access, vol. 7, pp. 24267-24281, 2017 https://doi:10.1109/access.2017.2763624.

[8] A. S. Abdelwahed, A. H. Zekry, H. L. Zayed, and A. M. Sayed, "Controlling electricity consumption at home smart home," Proc. - 2015 10th Int. Conf. Computer. Eng. Syst. ICCES 2015, pp. 49-54, 2016, https://doi: 10.1109/icces.2015.7393017.

[9] H. J. Jabir, J. Teh, D. Ishak, and H. Abunima, "Impacts of demand-side management on electrical power systems: A review," Energies, vol. 11, no. 5, 2018, doi: 10.3390/en11051050.

[10] N. Javaid, G. Hafeez, S. Iqbal, N. Alrajeh, M. S. Alabed, and M. Guizani, "Energy efficient integration of renewable energy sources in the smart grid for demand side management" IEEE Access, vol. 6, pp. 77077-77096, 2018, https://doi:10.1109/access.2018.2866461. 
[11]Z. Pooranian, J. H. Abawajy, P. Vinod, and M. Conti, "Scheduling distributed energy resource operation and daily power consumption for a smart building to optimize economic and environmental parameters," Energies, vol. 11, no. 6, 2018, https://doi:10.3390/en11061348.

[12] H. Shareef, M. S. Ahmed, A. Mohamed, and E. Al Hassan, "Review on home energy management system considering demand responses, smart technologies, and intelligent controllers,” IEEE Acce[5], vol. 6, pp. 24498-24509, 2018, https://doi:10.1109/ACCESS.2018.2831917.

[13] Yu-Hsiu Lin. (2018), "Design and Implementation of an IoT-Oriented Energy Management System Based on Non-Intrusive and Self-Organizing Neuro-Fuzzy Classication as an Electrical Energy Audit in Smart Homes", applied sciences, Vol 8 (12), 2337, 2018. https://doi:10.3390/app8122337.

[14] PIC18F2455/2550/4455/4550. Data Sheet, 2006 Microchip Technology Inc. 